\title{
Search reaction time for single targets in multiletter stimuli with brief visual displays*
}

\author{
J. T. TOWNSEND and R. N. ROOS \\ Purdue University, Lafayette, Indiana 47907
}

\begin{abstract}
The same set of Ss was run in an LT (single late target letter followed an earlier multiletter display) and an ET (single carly target preceded a later multiletter display) condition. On one-half the trials, the multiletter set included the target. and on the other half, the target was absent. The task of $S$ was 10 push the "yes" ("no") button if the target was present (absent), and reaction times were recorded. The most plausible processing model assumed that LI comparisons took place in a verbal-acoustic store and that ET comparisons took place in a visual store. It further assumed that processing within these stores was self-terminating, with rates that differed on "same" and "different" comparisons and which changed as the multiletter set increased. Classes of serial and parallel models that are falsified or supported by the present and similar data are discussed.
\end{abstract}

Questions posed about the processing involved in scanning of displays or immediate memory can be summarized roughly as dealing with processing capacity and its distribution in time and space (see, e.g., Gardner, 1970: Townsend. 1971). Thus, in tasks where S must process inure than one symbol, processing can be described as serial (one at a time) or parallel (simultaneous), or perhaps hybrid (neither). In addition, assumptions about S's processing capacity and efficiency (e.g.. limited-vs unlimited-capacity systems) result in predictions for probability correct and/or reaction time (RT) as functions of such variables as display time and number of symbols to be processed. If it is not necessary that all symbols in the display be processed in order to perform accurately. then whether $S$ processes all the symbols (exhaustive) or stops when sufficient information has been acquired (self-terminating) is also a pertinent theoretical issue.

Estes and Taylor (1964) give an experimental paradigm which effectively lessens the load on primary memory and in which behavior can be described in terms of the above characteristics. In this method, S must decide on each trial which of two signal (target) symbols is present in an array of randomly arranged noise symbols. This paradigm has been used primarily under degraded stimulus conditions and with consequent high error rates. Of interest here are two later techniques that are logically related to the detection paradigm but that employ high-accuracy conditions. These have been referred to as visual search and memory search designs.

In the typical visual search experiment (e.g., Atkinson, Holmgren. \& Juola. 1969). Ss are presented

\footnotetext{
*The authors would like to thank Dennis Mohrman and Donna Johnson for helping to prepare and run these experiments. Wichael Richards for computer prograniming assistance. and Donna Lucas for aid with numerical calculations and ty ping. Part of the work on this manusiripe was completed while the first author was Visiting Associate Professor at the laboratory of II. k. Estes at the Rockefeller Liniversity under tile support of Grant Gll16735 trom the National Institute for General Medical Sclences. Ile are indebied to H. Leeth. W. K. Estes. J. L. Holmgren. and J. S. Lappin for a number of help?ul remarks on an earlicr version of this manuscript.
}

with a single memory letter $(M=1)$, which is then followed by a variable-letter display $(D=1-5)$. The $S$ 's task is to search the display and give a "yes" (positive, + ) response if the memory letter is present and a "no" (negative, -.) response if it is not. Similarly, in a memury search study, Stemberg (1966) employed variable digit memory sets $(M=1-6)$, which were followed by single-digit displays $(D=1)$. S's task here was the same as above, except that this time the memory set was searched for the presence or absence of the display symbol. The major dependent variable in both paradigms has been RT. It has been pointed out (Wingfield \& Bolt, 1970) that "visual search" and "memory search" are difficult to distinguish, other than operationally. Indeed, for $D=M=1$, these tasks are equivalent, and statements about internal and external symbol codes used in the search process should be distinguished from the tasks per se. To this end, visual search and memory search designs will be referred to as early-target (ET) and late-target (LT) designs, respectively.

In the Atkinson et al ET study, both + and - RTs were found to be linearly increasing functions of $D$. with the -- function showing some early upward concavity. Although these functions had similar slopes. the intercept of the -- function was greater than that of the + . Also. it appeared that the serial position of the letter in the display had no strong effect on RT. In LT experiments, the results are similar. with RT now a function of M (e.g.. Sternberg, 1969). Studies utilizing an LT paradigm do not alway's yield homogeneous results (in some cases, probably due to different procedures, stimulus materials, etc.). however. and some notable exceptions to the usual results are that (a) the + RTs are sometimes nonlinear functions of $M$ (Briggs \& Swanson. 1969, 1970: Swanson \& Briggs, 1969). (b) differences in + and - RTs are not always observed (Sternberg. 1966: Wingfield \& Bolt. 1970: Wingfield \& Branca. 1970). (c) some investigators have found RT 10 be affected by the serial position of the target symbol in the memory set (Klatzky \& Atkinson. 1970: Klatzky. Juola. \& Atkinson. 1971: Waaver. MiLaughlin. \& Brancal 1970: Morin. DeRusa \& Stuluz. 1967: Norman. 


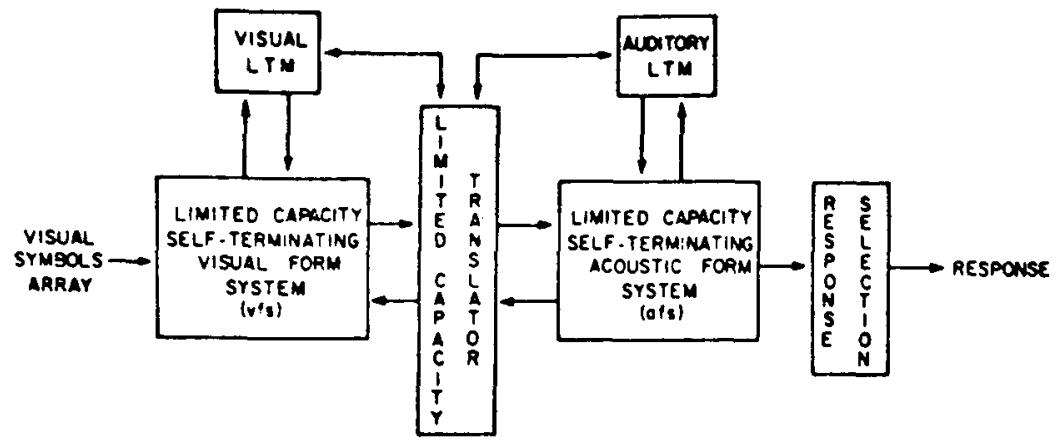

Fig. 1. Schematic of model for multisymbol search experiments with visual input.

1966: Corballis. 1967). and (d) the use of short intervals between presentation of memory set and target symbol reveals marked recency effects (Corballis, 1967: Morin, DeRosa. \& Stultz. 1967).

In the present study, the same Ss were run in LT and ET conditions and the results were used to help delineate a viable overall model of processing. The overall model will be presented in qualitative fashion below. The important issue of parallel vs serial scanning (in subsystems of the overall model) is treated in detail in the discussion. The failure of two representative parallel and serial models to account for both the - and the + RT functions leads to a mathematical investigation of classes of (self-terminating) parallel and serial models that can or cannot account for our and similar data.

Figure 1 shows a schematic of the overall model beginning with the input of visual material. Several of the mechanisms are quite familiar from other modeling attempts, and some have acquired substantial support: others are newer and/or more tenuous. The first hy pothetical system is a visual store (visual form system, vfs). which incluces the icon but also may be programmed from other systems with visual images or with feature (or symbol) alerting propensities. The latter facility forms part of its ability to process input symbolic material via matching with programmed simbolic material or (in other types of situations than the experiments here) by way of connections with visual long-term memory (LTM). The vfs is thought of as limited capacity in that when larger amounts of visual material are input, processing of the total aggregation consumes more time or is less accurate than when less material is presented.

The following mechanism, the translater, converts visual material into acoustic-verbal form, and vice versa. In ordinary linguistic behavior with visual input, it probably is closely tied in with access to visual and verbal-acoustic LTM. The translater is also conceived of as acting as the programming device from the acoustic channels to the vfs. We think it is limited capacity, requiring more time to translate longer strings of symbolic material, holding syntactic and semantic variables constant. It may also be serial for "unrelated" linguistic items.

The acoustic-verbal form system (afs) may be described in a fashion analogous to that of the vfs. It contains the traditional acoustic immediate memory and also the short-term iconic storage properties associated with auditory input. Whether scanning in ET or LT experiments is serial, parallel, or hybrid has not been determined with certainty (and in fact appears to be more subtle than earlier seemed likely: see, e.g., Townsend, 1972), and so we defer this question until the discussion.

The response selection system acts on the information that has accrued in the preceding charnels and chooses an appropriate response. Finally, we hypothesize that comparison times in afs and vfs take approximately the same time (under appropriate stimulus conditions). Although certain of the above mechanisms might tentatively be further broken down, the present delineation is sufficient for the experiments to be reported.

Witnin the context of this model, the case for limited-capacity systems both for vfs and for afs is very strong, associated as they are with increasing RT functions and decreasing accuracy functions of number of symbols presented (e.g., Estes \& Taylor, 1964; Sternberg, 1966: Atkinson, Holmgren, \& Juola, 1969). ${ }^{1}$ Knowledge about the capacity of the translator is much weaker. There are some experiments, however, that suggest limitations on its capacity (e.g., Klemmer, 1964: Pollack \& Johnson, 1965). It appears that it is not yet known precisely how much loss in information from that available in the icon (Sperling, 1960) is due to vfs processing and translation and how much to limitations in afs (i.e., in immediate memory).

The lack of strong serial position effects in Atkinson, Holmgren, and Juola's (1969) data is suggestive of exhaustive processing, but could also be due to a uniform prciessing path distribution in a serial system or a uniform distribution of attention in a parallel system. Further, the equality of slopes they and others have found in the + and - RT functions may be due to a dual processing mechanism (Bamber, 1969). Similarly, the equivalence of Sternberg's (1966) curves, although perhaps associated with exhaustive processing, could also follow from differential processing of + and - displays. Serial position information was not presented in the latter study. The notion of dual processing will be viewed in detail in the discussion and alternative possibilities considered. 
We shall provisionally suppose that both vfs and afs are basically self-terminating on the basis of spatial and serial position effects in ET (e.g., Estes \& Taylor, 1966) and LT studies (e.g., Klatzky et al, 1971), respectively, and an effort will be made to enhance such effects in the present work. Predictions concerning the similarity of ET and LT results now follow.

First, the (limited-capacity afs and vfs) model predicts increasing RT curves as functions of number of symbols presented; it rakes longer to make comparisons of the target with a greater number of nontarget symbols. This prediction differs from those of most previous investigators in putting the cause of increasing RT functions with the limited-capacity nature of the systems rather than with alleged seriality. Secondly, the supposition that comparisons related to vfs and afs are basically capable of self-termination suggests that instructions might influence the appearance of serial position effects. Their appearance would, in turn, support the concept of some optional control of distribution of attention on the part of Ss, since, as noted above, other studies have not always found serial position effects.

We next discuss the possible effects to be associated with the limited-capacity nature of the translator, since the operation of the translator is critical in determining how the ET and LT results compare with one another. There are several alternatives to consider.

The first assumes that in the ET condition, the second display symbols are encoded into afs and compared with the target and that in the LT condition, the early information is put in vfs after the target display appears and compared visually with the target. Comparison times in the two stores and translation time between the stores are assumed to be similar, and hence the RT functions are similar. We probably can summarily rule out this model as too unrealistic, since it assumes that a late multiletter display is compared acoustically with the target and vice versa for an early multiletter display.

A second alternative assumes that the ET processing involves holding (or inserting) the target (or pertinent features of the target) in vfs and making the comparison in vfs on appearance of the second display. In the LI condition, it postulates that the information from the first display has been placed in vfs before the appearance of the target and that comparison takes place in vfs. The similarity of RT functions follows. Since the display is on for a relatively long time in these experiments, such visual comparisons do not necessarily have to involve extraction of all of the information from the second display in the ET condition before comparison begins. Although more plausible than the first model, the assumption that several symbols can be effectively stored in the limited-capacity vfs after translation from afs during the short duration between stimulus presentations seems untenable, especially when these symbols will change on every trial in the present experiment.
The third possibility posits that $S$ translates visual information to afs in the LT condition for subsequent afs comparisons and stores the target in vfs in the ET condition for a visual type of comparison. Since comparison rates in the two types of stores are assumed to be similar, RT will be similar in the ET and LT conditions. This last model seems the most plausible of the three.

Aside from the possible failure of the assumption that comparison rates are similar in vfs and afs, there are two main hypotheses that predict differences in RT functions. The first assumes that ET comparisons take place in vfs and that in LT the symbols from the first display are translated to vfs after the second display is presented. This model predicts that the LT RTs will be larger in general than the ET RTs and will show greater increases in RT as a function of $M$ if the translation mechanism is a limited-capacity system. The second, analogous model assumes that comparisons take place in afs for both ET and LT conditions and that the ET condition produces longer RTs, since it involves more translation time than does the LT condition. The rate of increase of RT is also predicted to be greater in the ET than in the LT condition.

If the RT functions in the ET and LT conditions turn out to be similar in overall magnitude and general rates of increase, then either the translator is unlimited capacity (which seems improbable) and comparison rates in vfs and afs are similar or one of the first three alternative hypotheses gains support. If the RT functions substantially differently, then either the comparison times are quite different in ET and LT conditions or one of the last two hypothetical models acquires support.

\section{METHOD}

\section{Subjects}

Three naive undergraduates at Purdue. two female, served as Ss. All had 20/20 vision, corrected or uncorrected.

\section{Stimuli}

Stimuli were horizontal lists of from one to five English consonants, with no delimiters at the ends of the stimuli. Each i lisi was prepared by photographing white $1^{1 / 4}$-in. precut Gothic letters (by Duro, Chicago). which had been placed against a black background. (These colors were reversed by the high-contrast copy film.) A total of $1402 \times 2$ in. slides were made. including 20 single-letter slides and 30 slides each for stimuli of two to five letters. Selection of letters for each array was random. with the restriction that each consonant appeared only once per slide and that each letter appeared at least once in each serial position for each stimulus list length.

\section{Apparatus}

Slides were presented by a kodak Curousal R.t.950 projestor I variable-density filter was placed $t$ in. in front of the proficior to attenuate brightness. Images lacte projected on a 1 hics Plexiglas screan to in. fron the progecior. The sereen had fold

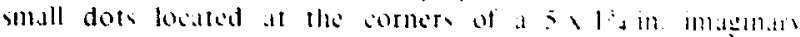

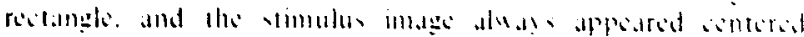




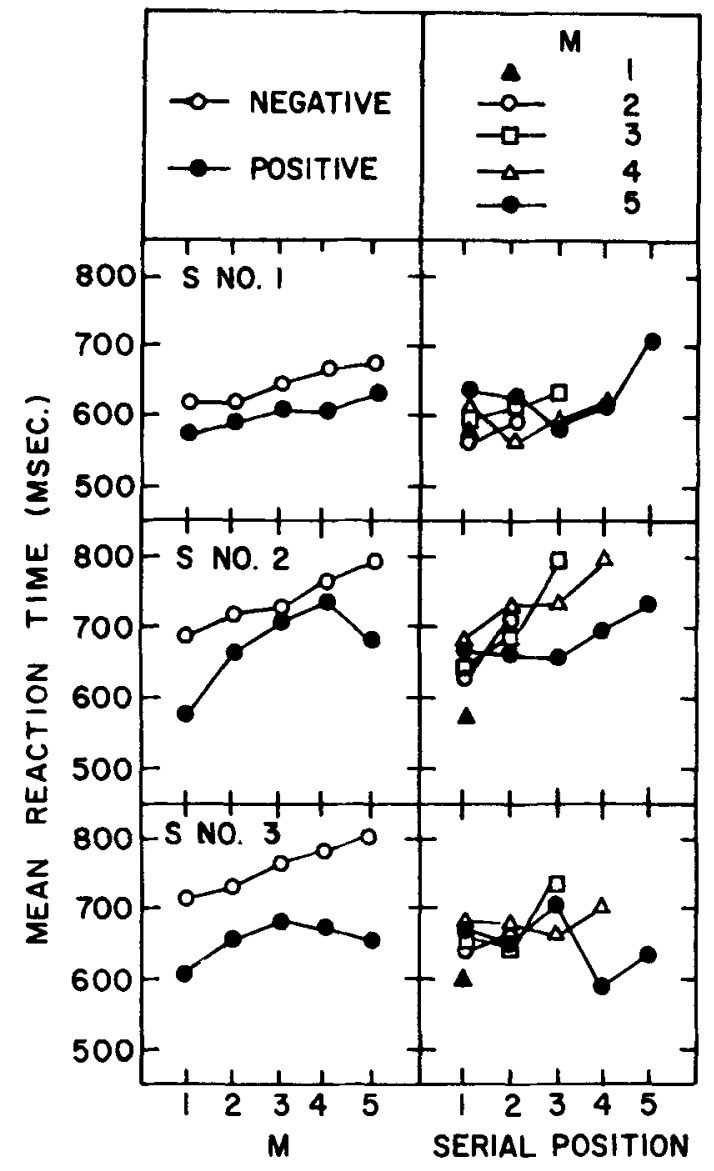

Fig. 2. Mean RT of positive and negative responses as a function of the number of letters in memory (left panel) and mean RT of positive responses as a function of the serial position of the target letter in the memory set (right panel) for the LT data.

within this rectangle. On the other side of the screen was a Panasonic TV camera (WV-220P), which communicated the stimulus to a Panasonic CCTV monitor (TR-900IM).

Ss were seated in front of a booth in an adjacent laboratory room. The booth was equipped with a Lafayette Instrument response panel (No. 12900) and a fixed viewing mask which served to hold the S's head steady. The TV monitor, whose knobs and halo were masked so that only a $3 \times 5$ in. white visual area could be seen, was inside the booth and 29 in. from the viewing mask. Small red and yellow lamps were mounted above the monitor. Centered within the visual field were the four dots of the imaginary rectangle, which now subtended a visual angle of $5.5 \times 2.0$ deg. A five-letter display, as it appeared on the screen, was $5.5 \times 0.75 \mathrm{deg}$ of visual angle. Brightness of pre-post and stimulus fields was 8.1 and $16.9 \mathrm{fL}$, respectively, as measured by a Gamma Scientific photometer 2020 .

All trial events were controlled by means of a Lafayette Data System 12800 . The appropriate slides were selected by reading in punched paper tape. Exposure duration was regulated by a shutter placed in front of the projector. The shutter consisted of a circular section of aluminum, which was raised and lowered by a Ledex rotary solenoid (No. H-2419-031). Stimuli, responses, and reaction times (accurate to $\pm 1 \mathrm{msec}$ ) were recorded on punched paper tape. Reaction times were measured as the time between onset of stimulus and depression of a response button. Ss wore headphones and heard white noise, adjusted to a comfortable level, to mask possible cues from the slide selection mechanism, throughout each experimental session.

Trial events consisted of (a) a red warning light, (b) presentation of memory slide for $2 \mathrm{sec}$, (c) a 3 -sec delay, (d) presentation of second slide for $400 \mathrm{msec}$, (e) S's response, (f) a yellow feedback light if the response was correct, and

(g) $1.8 \mathrm{sec}$ before the next trial.

\section{Design}

The experiment was run in two parts. In the first (LT) part, Ss saw the variable set slide (1-5 letters) before they saw the test element slide (1 letter). This was reversed for the second (ET) part. There were 11 sessions in each part, and of those, the last 10 were used for data collection. Each session had 250 trials, in which conditions were randomized with the following restrictions: (a) + and - trials occurred equally of ten, (b) each list length occurred equally of ten within each response type, and (c) the test letter appeared about the same number of times in each serial position of each list length.

\section{Procedure}

On the first day, Ss were read instructions which described the procedure to be followed. They were told to rest the index finger of their dominant hands on the center button of the response panel, and that the button to the left registered a "yes" response and the one to the right registered a "no" (the movement required was about $7 / 8$ in.). Ss were also instructed to "scan" all visual displays from left to right and to respond quickly but make as few errors as possible

In each session, there was a 5-min dark-adaptation period, 20 practice trials, a brief delay, then 250 data-collection trials. The latter were interrupted for about 5 min at Trial 125 so that slide trays could be changed. A complete experimental session lasted approximately an hour, and each $S$ was run at the same time each day until the experiment was finished.

\section{RESULTS AND DISCUSSION}

The response error rate was found to be approximately $1.8 \%$ in both parts of the experiment, and reaction times were longer than $2 \mathrm{sec}$ for less than $1 \%$ of all responses. These trials were eliminated from further analysis.

Figure 2 shows mean RT as a function of $M$ and as a function of the serial position of the target letter in the memory set for each $\mathrm{S}$ in the LT experiment. RTs as a function of $\mathrm{D}$ and the serial position of the target letter in the display for the (same) Ss in the ET design are given in Fig. 3. These same data, averaged over Ss, are given in Fig. 4. The slopes and intercepts of the best-fitting straight lines through these functions, calculated by the method of least-squares deviations are (a) $586.4+18.0(\mathrm{M})$ and $646.8+21.6(\mathrm{M})$, and (b) 578.3 $+22.7(\mathrm{D})$ and $654.2+15.4(\mathrm{D})$ for + and - functions, respectively. If the point for $D=1$ is eliminated from the - RTs of the ET results, the best-fitting line through the remaining points is $635.4+20.1$ (D).

The first prediction of the overall model, that the RT curves would increase, was confirmed, suggesting limited-capacity processing in vfs and/or afs. The nonmonotonicity in the LT condition for two of the Ss was unexpected. After checking for such artifacts as higher error rates for larger memory sets and interstimulus duration being correlated with memory set, without success, we ran two more Ss in a LT 


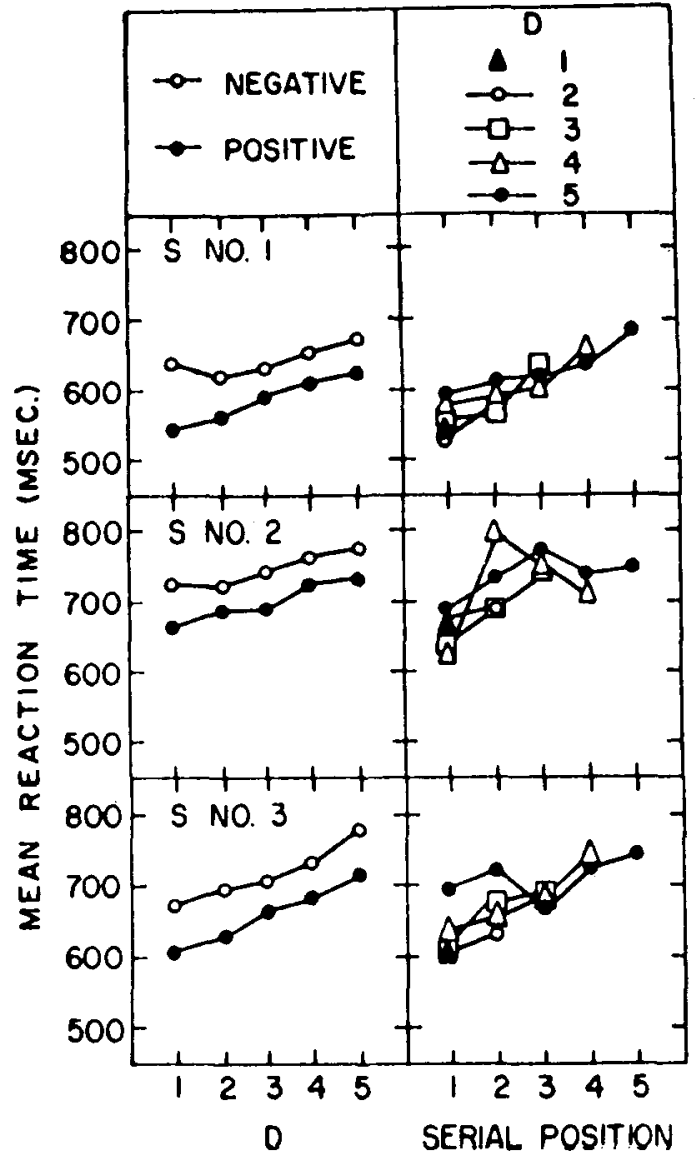

Fig. 3. Mean RT of positive and negative responses as a function of display size (left panel) and mean RT of positive responses as a function of the serial position of the target letter in the display (right panel) for the ET data.

condition. The results for these Ss are given in Fig. 5: again, one of the Ss' data shows a curvilinear effect. It has been suggested (e.g., Swanson \& Briggs, 1969) that curvilinearity in LT experiments may actually reflect a linear relationship of $\mathrm{RT}$ to central processing uncertainty. The fact that three of the five Ss' LT graphs reveal some decrease may mean that this hypothesis will not suffice to explain these effects. The Ss' spontaneous introspections did not reveal any differences in processing strategy that might underlie them. We are presently uncertain as to the origin of the nonlinearities.

With respect to other ET and LT characteristics, the overall magnitudes of the correct ET RTs (+RT = $648.37 \mathrm{msec},-\mathrm{RT}=701.19 \mathrm{msec}$ ) are quite close to those of the correct LT RTs (+RT $=640.71 \mathrm{msec}$, - RT $=704.11 \mathrm{msec}$ ) considering the average standard error was $5.32 \mathrm{msec}$. So, also, are the general amount of increase over $D$ and $M$ and the intercepts. The only salient exception is S 3 , whose - LT curve is definitely above his - ET curve. Interpretation of these statistics requires nuting the learning effects which were quite noticeable in the LT results (with an overall mean RT going from 728 in Sessions 1, 2 10600 msec in Sessions 9. 10). bur nut in the ET resulis (1). 2 nd sission mean of 0609 mse 10 a 9 (h) 10 th session mean of 675 : 11 sec)

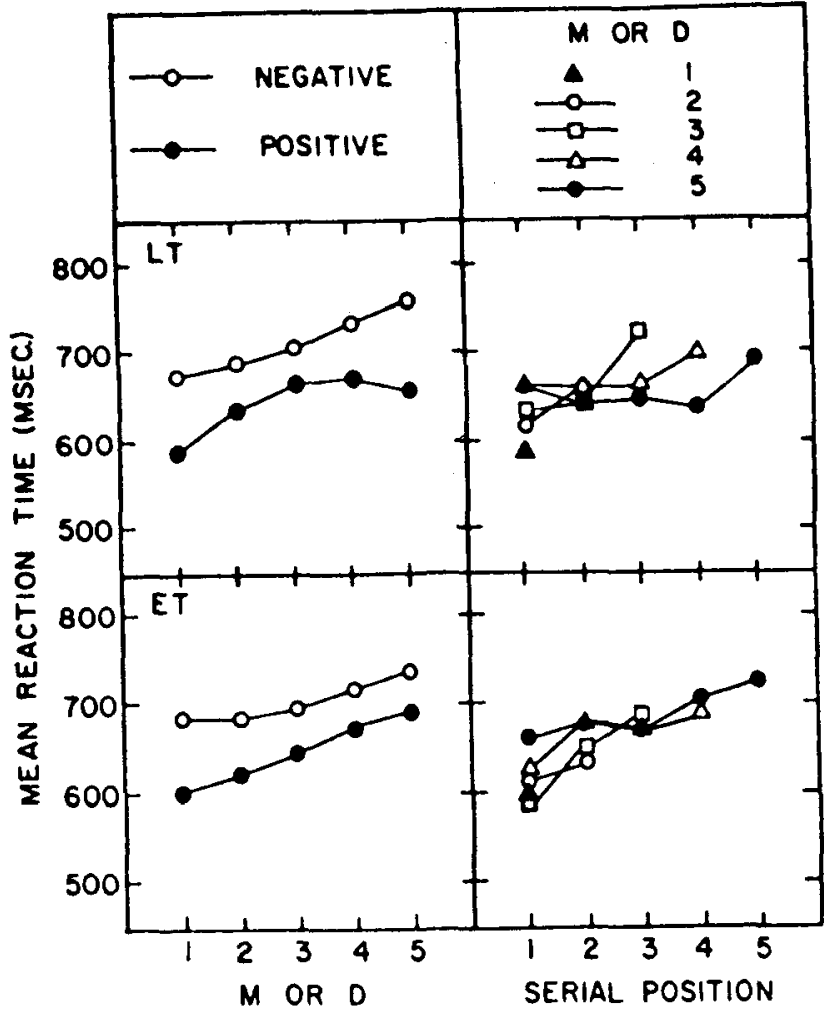

Fig. 4. Mean RT of positive and negative responses as a function of the number of letters in memory or in the display (left panel) and mean RT of positive responses as a function of the serial position of the target letter (right panel) averaged over Ss for the LT and ET data.

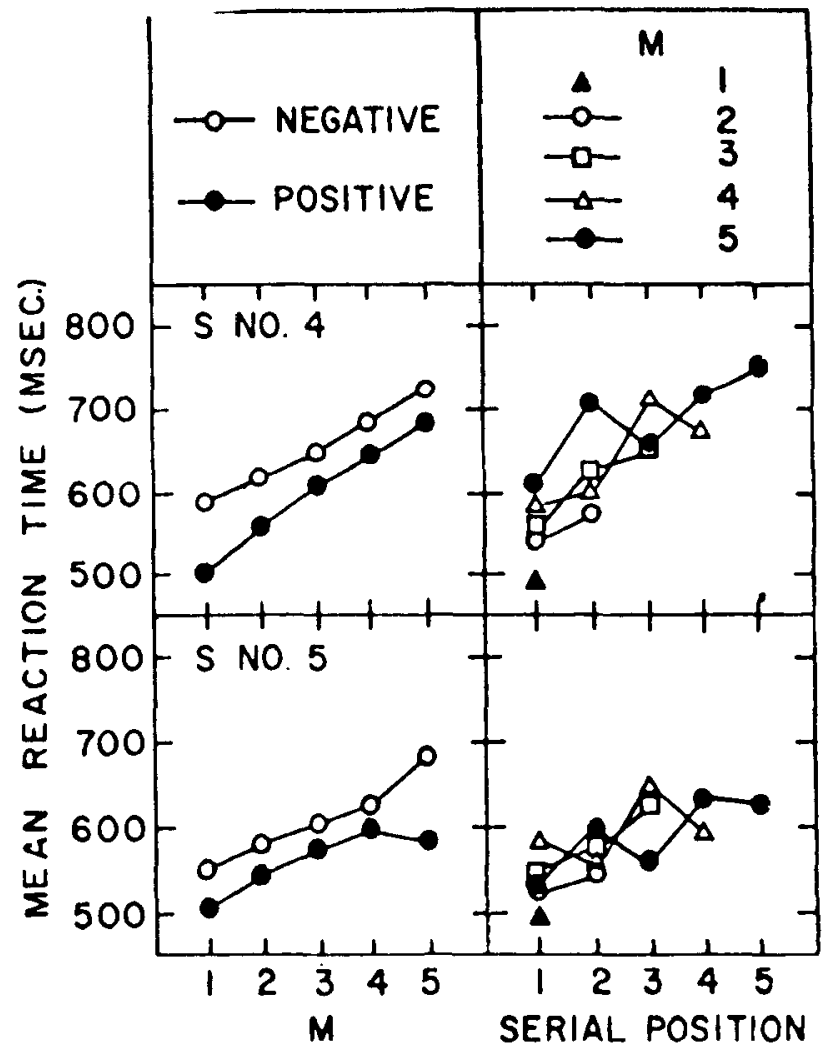

Fig. 5. Mean RT of positive and negative responses as a function of the number of letters in memory (left pancl) and mean RT of positive responses as a function of the target litter in the memory set (right panel) for th: two additional Ss run in the LI design. 
Further, the LT mean RT was still decreasing and was lower than the last ET mean RT and the learning appeared to affect mainly the intercept rather than slope. The latter facts plus the finding that the overall performances were so close, with the LT learning curves intersecting the flatter ET curves, argue for support of the third hypothesis of the overall model on translator functioning: comparisons in the ET condition take place in vfs, comparisons in the LT condition take place in afs, and comparisons can proceed on the average at similar rates in afs and vfs under appropriate conditions of stimuli, apparatus, and learning. The learning differences between ET and LT conditions are intriguing and may deserve further study. The qualitative shape (as opposed to general increases and magnitude) differences in the + RT function between the ET and LT conditions for two of the Ss also suggest that the comparisons do not take place in the same store. The fourth possible explanation of comparable curves in the two conditions, involving a translator of unlimited capacity, seems quite unlikely, as do the first two of the three explanations involving a limited-capacity translator, but cannot be definitely ruled out at present.

The appearance of serial position effects, strongest in the ET condition but also present in the LT condition (especially in S2's and S4's data), appears to give confirmatory evidence for self-termination of processing on + trials in vfs and afs. There is the possibility that the (left-to-right) serial position effects were due to some kind of response compatibility effect since the "Icft" response denoted location of a target. Further work will be required to resolve this issuc, but the fact that the Atkinson et al Group 1 (with a similar stimulus responsc mapping) results showed sucti negligible serial position effects relative to Group 2's (with a left-no, right-yes mapping) is a possible argument against that hypothesis. Another possibility, that the Ss were fixating to the left of center in the second display, can affect only the speed or likelihood of processing the leftmost letters, not whether or not processing is exhaustive instead of self-terminating. Further, the fact that others have obtained evidence supportive of self-termination (e.g., Estes \& Wessel, 1966; Nickerson, 1966; Sternberg, 1967) in ET experiments (references for similar LT experiments were cited earlier) suggests that it should be accorded serious consideration. Also, since strong serial position effects were found here with left-to-right scan instructions, but not in the Atkinson et al (1969) study, the possibility arises that $S s$ may be capable of manipulating their distribution of attention, at least within certain spatial and temporal limits. It also appears unlikely that $S s$ are innately self-terminating or exhaustive processors or asymmetric vs symmetric distributors of attention; however, it may be that they come, through experience, to function in certain characteristic ways.

A recent study, which varied ET and LT set sizes simultaneously (Burrows \& Murdock, 1969), was presented in a manner somewhat difficult to compare with those here, but two germane points may be noted. First, stronger serial position effects were found in the ET ("memory" set or first display) than in the second display [which is in contrast with Sternberg's (1967) results supporting exhaustive early display seanning and self-terminating late display scanningl, and secondly, the effect of number of items in the first display appeared to be stronger (steeper slope) than in the second display. However, the fact that 1-3 items were employed in the first display but 3-6 were used in the second display makes the second point depend on the assumption of extrapolated linearity. In any case, the first, but not the second, agrees with the present data. The reason for the latter disparity with the present results may be partly due to the comparatively high luminance $(90 \mathrm{lL})$ of their second display (the first display was presented auditorially) and the use of dark pre-and postexposure ficlds. Further, we are not arguing that processing rates for ET and LT comparisons will alwavs be the same but only that it is possible to find conditions under which they are similar (as during the middle sessions of the LT and ET conditions in the present experiment).

Although the details differ somewhat, lhe individual RT curves tend to increase at about the same rate and the scrial position curves tend to show an increase in RT from Position 1 to Position 5, as the individual and the average results in Figs. 2, 3, and 4 indicate. In order to see if the overall characteristics of behavior in this experiment contained any implications for the parallel-serial question, or information as to whether processing involves unary vs dual mechanisms, a parallel and serial model were litted to the average + RT curves in the two conditions for the three $S s$ that were employed in both conditions, and then the parameter estimates used to predict the average - RT data. ${ }^{2}$

Unfortunately, as previously shown (Townsend, 1972), the present types of data (as well as most previous data with which we are familiar) do not casily permit firm tests as to parallelity vs seriality. However, it may be of value to see how a parallel model with independent processing compares with a scrial model that assumes equal processing rates for the various serial positions. ${ }^{3}$

Both models assume self-termination on + trials, both are based on exponential processing of individual symbols, and both arc capable of reflecting scrial position effects. The serial model posits a nondegenerate probability distribution over the set of possible processing paths through the symbols; it is the possibility that some paths are preferred over others that gives the requisite structure for serial position effects. The independent parallel model yields serial position effects via different processing rates on the different positions. Both models allow for different residual latency components and possess the same number of parameters. 4 A brief mathematical presentation of the models is given in Appendix $A$. 
A perfect fit for the parallel model (as well as for those serial models that are equivalent to it) to the + RTs is guaranteed, but the serial model is open to falsification (see Appendix A). However, the serial model came within \pm 1 standard error for all except one serial position point for the data reported here; hence, the + RT predictions are drawn coincident with the experimental points in Fig. 6 . Thus, these + RT data do not, for all practical purposes, discriminate between an independent parallel model and the present constrained-path mixed serial model.

As suggested from the linearity in the $+E T$ results, the estimated processing rates for the serial model (the $u_{n} s$, Apjocriutix A) were almost equal for each value of $n$ (range is .0214 to .0225). This was not true, of course, in the $+L T$ results. Interestingly, the sum of the parallel processing rates

$$
\left(\sum_{i=1}^{n} v_{n, i}, \text { Appendix A }\right)
$$

were also fairly constant across $n$, as shown in Table 1 , for the ET data, but not for the LT data. This is supportive of a constant amount of processing capacity for the ET condition, within the context of the parallel model, which can be spread across the symbols in a not necessarily uniform manner. The primary difference between the present parallel model and those parallel models that are acruaily equivalent to the present serial model is that the former does not assume the capability to reallocate the processing capacity from completed symbols to uncompleted symbols within a single trial.

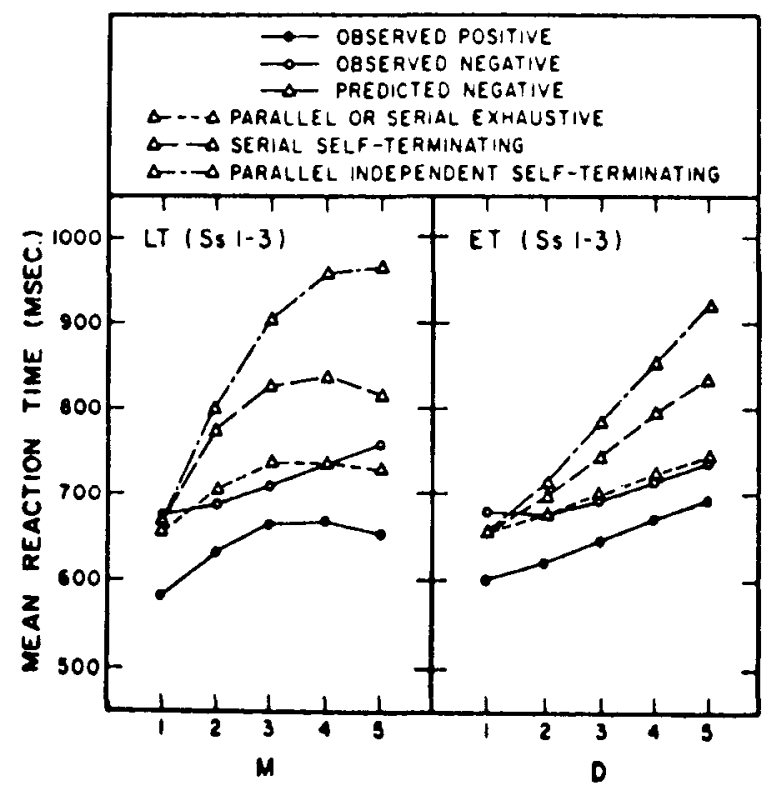

Fig. 6. Observed positive and negative mean $\mathrm{RT}$ as a function of $M$ and $D$ for the three $S s$ used in both ET and LT studies, and predicted parallel and serial mean negative RT, assuming that the positive RTs were generated by processes which were either crlaustive or self-terminating.
Table 1

Sums of Serial Position Processing Rates of Parallel Model for ET and LT Data as a Function of the Number of Letters to be Processed

\begin{tabular}{|c|c|c|c|}
\hline $\mathrm{N}$ & ET & $\sum_{i=1}^{n} v_{n, i}$ & LT \\
\hline 1 & .0215 & & .0097 \\
\hline 2 & .0314 & & .0131 \\
\hline 3 & .0418 & & .0168 \\
\hline 4 & .0391 & & .0211 \\
\hline 5 & .0376 & & .0285 \\
\hline
\end{tabular}

The predictions of the - RT curves are also shown in Fig. 6. It is apparent that neither the serial nor the parallel self-terminating model even comes close to predicting the observed points. As is evident in the figure, either a serial or a parallel model with exhaustive processing and different residual latency components for ,+- trials can come relatively close (ignoring the concave-down appearance of the + LT case). However, exhaustive models meet severe problems in handling various types of item-position effects. Bamber (1969) found a similar problem with his data from a same-different experiment using letters as stimuli. He proposed that instead of there being one process which determines whether or not the stimulus matches a memory symbol, there might exist two distinct comparison processes. One, the identity reporter, would be fast and emit signals for "same" responses only. The other, a serial processor, would be slow and emit signals for both "same" and "different" responses. These components apparently function simultaneously in a semiautonomous fashion. Although such mechanisms. which apparently refer to two separate measuring components, are not out of the question, we are reluctant to drop the more parsimonious single component processing mechanism until more evidence is brought against it. An alternative idea is that the same (self-terminating) measuring or processing mechanism might nevertheless process + and - symbols at different rates. We shall pursue this alternative below.

One fact about multisymbol search experiments is quite clear: it is quite rare to find that the - RT functions possess exactly twice the slope of the + RT functions (exceptions are Clifton \& Birenbaum, 1970: and possibly Burrows \& Murdock. 1969), although Iinearity is common. Most studies in the literature do ieport + RT slopes that are less than or equal to the RT slopes, and equality of slopes is frequently reported. These findings lead us to investigate the implications that equal slopes, such as found in the present data (and others, e.g., Atkinson et al. 1969: Sternberg, 1966). hold for unary processing mechanisms with different rates for + and - comparisons. Murdock (1971) is able to obtain approximately equal \pm RT slopes in a parallel model. as do Theois. Smith. Haviland. Traupman.\& Moy (1973) in a serial model. both assumine selftermination. Tha aresent stuly is appatently the first anderio 
examination of this question in large classes of serial and parallel models.

It is well known that a self-terminating serial model with a constant processing rate predicts a $2: 1$ ratio of RT to + RT slopes, but how many other more general serial models cannot predict equal slopes is not known. We proceed to show that there is a quite large class of serial models that cannot predict equal slopes and then investigate some violations of the attendant assumptions in a simple serial model that can predict equal slopes. $\mathbf{A}$ parallel model that also can predict equal slopes is then developed and compared with its serial counterpart.

Letting $t_{i j}$ be the random variable that represents processing time on the $\mathrm{i}^{\text {th }}$ symbol when it is processed $j$ th in order and $E\left(t_{i j}\right)$ be the expectation (mean) of that random variable, we characterize the class of serial systems that cannot predict equal slopes for $+\mathrm{RT}$ and RT by the following assumptions:

(1) The system is self-terminating with a probability distribution on processing paths through the symbols, given by

$$
\mathrm{p}_{\mathrm{k}}, \mathrm{k}=1,2,3, \cdots, \mathrm{n} !-1, \mathrm{n} !, \text { and } \sum_{\mathrm{k}=1}^{\mathrm{n} !} \mathrm{p}_{\mathrm{k}}=1 \text {. }
$$

(2) Equal average time increment assumption for + symbols:

$$
n \cdot E_{n}\left(t^{+}\right)=\sum_{k=1}^{n !} p_{k}\left[\sum_{i=1}^{n} E_{n}\left(t_{i,(k)}^{+}\right)\right]=n \cdot E_{1}\left(t^{+}\right),
$$

where $t_{i,(k)}^{+}$stands for the time taken to process the $i^{\text {th }}$ symbol in the display when its order is specified (exactly) by the $\mathrm{k}^{\text {th }}$ permutation in processing orders and it is the target $(+)$ symbol. $\mathrm{E}_{\mathrm{n}}\left(\mathrm{t}^{+}\right)$is the total average time to process the + symbol, averaged across serial position and processing order; it must not be confused with $\mathrm{RT}^{+}$(specifically, $\mathrm{RT}^{+}-\mathrm{t}_{0}^{+}$), which, of course, includes $t_{\mathbf{i j}}$ components. Note that $1 / \mathrm{n}$ gives the uniform probability that the E selects the $\mathrm{i}^{\text {th }}$ position to put the + symbol. The assumption then says that if the components are neglected, the average + processing times remain constant. Two special cases that predict this are (a) for any fixed $k$, assuming the

$$
\sum_{i=1}^{n} E_{n}\left(t_{i,(k)}^{+}\right)=n E_{1}\left(t^{+}\right)
$$

i.e., the sum of + times for any given $\mathrm{k}$ is $\mathrm{n}$ times as large as the time necessary to process the + symbol when $n=$ 1. This type of intuition gives the rationale for the "increment" in the heading; (b) simply let $\mathrm{E}_{\mathrm{n}}\left(\mathrm{t}_{\mathrm{i},(\mathrm{k})}^{+}\right)=$ $\mathrm{E}_{1}\left(\mathrm{t}^{+}\right), \mathrm{i}=1,2, \cdots, \mathrm{n}$ and $\mathrm{k}=1,2, \cdots, \mathrm{n}$; this latter is the traditional serial model.

(3) Equal average time increment for - symbols:

$$
n \cdot E_{n}\left(t^{-}\right)=\sum_{k=1}^{n !} p_{k}\left[\sum_{i=1}^{n} E_{n}\left(t_{i,(k)}^{-}\right)\right]=n E_{1}\left(t^{-}\right),
$$

where the notation is as above except that since no + symbol is included on - trials, $\mathrm{nE}_{n}\left(\mathrm{t}^{-}\right)=\mathrm{RT}_{\mathrm{n}}^{-}-\mathrm{t}_{0}^{-}$, i.e., the average time for processing on - trials is $n$ times that for a one-symbol condition $(n=1)$.

Note that this class of systems is quite large in that processing times on different serial positions can differ, the time for a given serial position can change as $\mathrm{n}$ varies, and times for target symbols can be different from those for nontarget symbols. And, while the - RT function (of $n$ ) is predicted to be a straight line, the + RT function is not necessarily straight. It is shown in Appendix B that the + RT function cannot be straight with slope equal to that of the - RT function. ${ }^{5}$

The derivation in Appendix B is based on models whose most salient (and almost the only) common characteristic is that as $n$ increases, the average total amount of time spent on + or - symbols increases proportionately. This characteristic is a generalization of the usual serial assumption that the processing rate is constant over everything. We might expect, then, that if there exist serial, self-terminating models that can predict exactly equal slopes, they must somehow violate this characterizing assumption. ${ }^{6}$

By investigating the constraints imposed on a simple serial model when equality of slopes is maintained, we verify this supposition and gain some intuition about how this violation is evidenced mathematically. Let $u_{n}^{-}=$ the exponential processing rate for - symbols and $u_{n}^{+}=$ the exponential processing rate for + symbols for displays of size $n$. Then the average total time to make a positive match, i.e., to locate the + symbol, is given by

$$
\mathrm{E}_{\mathrm{n}}\left(\mathrm{t}^{+}\right)=\left(\frac{\mathrm{n}+1}{2}-1\right) \frac{1}{\mathrm{u}_{\mathrm{n}}^{-}}+\frac{1}{\mathrm{u}_{\mathrm{n}}^{+}},
$$

and the average total time to process all $\mathbf{n}-$ symbols on - trials is given by

$$
E_{n}\left(t^{-}\right)=\frac{n}{u_{n}^{-}}
$$

It is instructive to analyze this model under the assumption that the constant difference between the + RT and - RT functions is due to processing, i.e.,

$$
\mathrm{RT}_{\mathrm{n}}^{+}=\left(\frac{\mathrm{n}+1}{2}-1\right) \frac{1}{\mathrm{u}_{\mathrm{n}}^{-}}+\frac{1}{\mathrm{u}_{\mathrm{n}}^{+}}+\mathrm{t}_{0}=\mathrm{kn}+\mathrm{t}_{0}
$$

and

$$
R T_{n}=\frac{n}{u_{n}^{-}}+t_{0}=k n+t_{0}+r, r, k>0,
$$


where $t_{0}$ is the sum of common residual times and $r$ is some additional constant time necessary to frocess symbols. By employing the starting values for $n=1$ to obtain $\mathrm{k}=1 / \mathrm{u}_{1}^{+}$and $\mathrm{r}=\left(1 / \mathrm{u}_{1}^{-}\right)-\left(1 / \mathrm{u}_{1}^{+}\right)$, we are able to solve the above equations for $u_{n}^{+}$and $u_{n}^{-}$:

$$
u_{n}^{-}=\frac{1}{\frac{1}{u_{1}^{+}}+\left(\frac{1}{u_{1}^{-}}-\frac{1}{u_{1}^{+}}\right) \frac{1}{n}}
$$

and

$$
u_{n}^{+}=\frac{1}{\frac{1}{n} \cdot \frac{1}{2}\left(\frac{1}{u_{1}^{-}}-\frac{1}{u_{1}^{+}}\right)+\frac{n}{2 u_{1}^{+}}+\frac{1}{u_{1}^{+}}-\frac{1}{2 u_{1}^{-}}} .
$$

Taking finite differences or approximating with derivatives indicates that $u_{n}^{-}$increases as a function of $n$ to the value $u_{1}^{+}$, while $u_{n}^{+}$can show some fluctuation for small $n$ for certain $u_{1}^{-}, u_{1}^{+}$values but always ultimately decreases. In any case, since

$$
u_{n}^{+}=\frac{1}{k n-\left(\frac{n+1}{2}-1\right) \frac{1}{u_{n}^{-}}} .
$$

11 follows that the increase in $u_{n}^{-}$affects $u_{n}^{+}$by tending 1o increase $i_{t}$ as it $\left(u_{n}^{-}\right)$increases. Also, it appears that $u_{n}^{+} / u_{n}^{-}$decreases for all but "small" values of $n$. This decrease in $u_{n}^{+}$relative to $u_{n}^{-}$can be seen even more clearly when it is assumed that the constant difference between the +RT and $-\mathrm{RT}$ curves is completely due to the residual time components, for example, a response bias. In this case, $u_{n}^{-}$remains constant and $u_{n}^{+}$decreases monotunically. It is simple to analyze and its development is lett to the reader.

We take special note that the assumptions in the serial models that could not predict equal slopes are not met here. For example, when $n=2$.

$$
\begin{aligned}
n_{n} E_{n}\left(t^{+}\right) & =2 E_{2}\left(t^{+}\right)=p \frac{1}{u_{2}^{+}}+(1-p) \frac{1}{u_{2}^{+}}=\frac{1}{u_{2}^{+}} \\
& =\frac{1}{4}\left(\frac{1}{u_{1}^{-}}-\frac{1}{u_{1}^{+}}\right)+\frac{2}{u_{1}^{+}}-\frac{1}{2 u_{1}^{-}} \\
& =\frac{7}{4 u_{1}^{+}}-\frac{1}{4 u_{1}^{-}} \neq 2 \cdot E_{1}\left(t^{+}\right)=\frac{2}{u_{1}^{+}} .
\end{aligned}
$$

Violation of the constraints on - processing times may be similarly demonstrated.

To proceed with similar developmenis for parallel models, we again assume exponential processing on the individual symbols and that processing on + trials is self-terminating. It is turther assumed that processing on the different symbols is independent and takes place al the same rate for different serbil positions. The latter restriction is not necessary but leads to considerable simplification of the mathematics and explication of processing mechanisms. Let $\mathrm{v}_{\mathrm{n}}^{+}=$the processing rate for the + symbols when $n$ symbols are presented and $v_{n}^{-}=$ the processing rate for the - symbols when $n$ symbols are presented. Then, it follows from our assumptions that the expected processing times on + and - trials, respectively, are

$$
E_{n}\left(t^{+}\right)=\frac{1}{v_{n}^{+}} \text {and } E_{n}\left(t^{-}\right)=\frac{1}{v_{n}^{-}} \sum_{i=1}^{n} \frac{1}{i} \text {. }
$$

The intuition on the + expression is that since processing on the individual symbols is independent and of the rate $\left(v_{n}^{+}\right)$, the mean + processing time is just the reciprocal of the rate on the + symbol, namely $1 / \mathrm{v}_{\mathrm{n}}^{+}$. On the other hand, the - exhaustive processing can be broken down into $n$ stages: Stage 1 (no symbols completed) proceeds up until the first symbol is finished at a rate of $n v_{n}^{-}$(the rate to minimum completion time of $n$ exponentially distributed random variables), the second stage takes place up until the second symbol is completed at rate $(n-1) v_{n}^{-}$, and so on until only a single symbol remains at Stage $n$ and of course is completed with Rate $v_{n}^{-}$. The overall mean is then simply the sum of the reciprocals of the component rates:

$$
\sum_{i=1}^{n} \frac{1}{v_{n, i}^{-}}=\frac{1}{v_{n}^{-}} \sum_{i=1}^{n} \frac{1}{i}
$$

when the rates are homogeneous across serial positions.

Employing the same type of assumptions concerning $t_{0}$ (the residual time) and the rationale for the constant difference between + RT and - RT, we obtain the conditions for linearity and equality of slope,

$$
\mathrm{RT}^{-}=\frac{1}{v_{\mathrm{n}}^{-}} \sum_{\mathrm{i}=1}^{\mathrm{n}} \frac{1}{\mathrm{i}}+\mathrm{t}_{0}=\mathrm{kn}+\mathrm{t}_{0}+\mathrm{r}
$$

and

$$
\mathrm{RT}^{+}=\frac{1}{\mathrm{v}_{\mathrm{n}}^{+}}+\mathrm{t}_{0}=\mathrm{kn}+\mathrm{t}_{0}
$$

Solving these equations finds

$$
v_{n}^{+}=\frac{v_{1}^{+}}{n}
$$

and

$$
v_{n}=\frac{\sum_{i=1}^{n} \frac{1}{i}}{\frac{1}{v_{n}^{+}}+\left(\frac{1}{v_{n}^{-}}-\frac{1}{v_{n}^{+}}\right)}=\frac{\sum_{i=1}^{n} \frac{1}{i}}{\frac{n}{v_{i}^{+}}+\left(\frac{1}{v_{i}^{-}}-\frac{1}{v_{i}^{+}}\right)}
$$


where

$$
\mathrm{k}=\frac{1}{\mathrm{v}_{1}^{+}}, \mathrm{r}=\frac{1}{\mathrm{v}_{1}^{-}}-\frac{1}{\mathrm{v}_{1}^{+}}
$$

Here we assumed that $r$ was due to + or - symbol processing rather than other processes common to both. Taking finite differences for $n=1,2, \cdots$, of $\overline{v_{n}}$ shows that $v_{n}$ can at most increase for small $n$ but always eventually decreases; clearly, $v_{n}^{+}$always decreases and at a faster rate than $v_{n}^{-}$(that is, $v_{n}^{+} / v_{n}^{-}$decreases monotonically as a function of $n$ ). If we assume that $r$ is due to residual processes, the results are even simpler:

$$
v_{n}^{-}=\frac{v_{1} \sum_{i=1}^{n} \frac{1}{i}}{n}, v_{n}^{+}=\frac{v_{1}}{n},
$$

where $1 / k=v_{1}^{-}=v_{1}^{+}=v_{1}$, and both $v_{n}^{-}, v_{n}^{+}$decrease monotonically, but with $v_{n}^{+}$at the faster rate.

The reader may have noticed that the difference $r$ might have been relegated to the + rather than the comparisons. For that matter, qr $(0<q<1)$ might be ascribed to the - and $(1-q)$ r to the + process. The latter more esoteric case is excluded here because we do not feel enough is known empirically to presently justify its inclusion. The former is not shown since it implies that $k>r$, where $k$ is the slope and $r$ is the initial difference, $\mathrm{RT}_{1}-\mathrm{RT}_{1}^{+}$, and this is false for the presen $i$ data.

The possibility of different rates for + and - symbol comparisons and for different values of $n$ appears to be of more than mathematicak interest. Sternberg (1969) has produced evidence that the difference can be affected by manipulation of + and - trial frequencies, which gives support to the possibility that the difference is due to response bias in LT studies. The findings by Egeth, Jonides, and Wall (1972), Brand (1971), and others of the critical influence of the type of target and noise symbols on overall magnitude and rates of increase of RT curves are strongly suggestive that internal processing rates may vary accordingly, at least in ET experiments. The above four models that can predict equal slopes may have different implications for the underlying comparisons.

The two serial and parallel models that place the initial - RT and + RT difference in residual mechanisms each assume that the + and - rates are equal for $n=1$ but that the + rate then becomes increasingly slower relative to the - rate for larger $n$. The other two models, those that assume that the initial difference is due to the - rate being slower (smaller) than the + rate, can predict an increase or decrease in the ratio of + rates to - rates as a function of $n$, for small $n$. Using values for the + and - rates that approximate those found in the present study indicates that the latter serial model shows $u_{n}^{-}$as increasing for $n=1, \cdots, 5$, and $u_{n}^{+}$as decreasing over the same range and with $u_{n}^{-}>u_{n}^{+}$except for $n=5$. On the other hand, the latter parallel model shows $v_{n}^{-}$as increasing slightly, then decreasing, and $v_{n}^{+}$as steadily decreasing from $n=1, \cdots, 5$, and $v_{n}^{-}$becomes larger than $v_{n}^{+}$at $n=4$.

Some may be inclined to place a tentative advantage with the models that presume the initial RT (intercept) difference to lie in $t_{0}$ rather than in the rates themselves, for few "natural" mechanisms come to mind that would predict that individual symbols are processed more rapidly, the greater the number to be processed, even for small values of $n .^{7}$ With respect to the serial-parallel predictions, the serial model does not seem quite as compatible with the idea that its processing rates slow down for larger $n$. Nevertheless, if increasing $n$ means more information between the early symbols and late symbols must be employed in the comparisons, then either might make such predictions. When slower + than - comparison times are predicted, a possible implication is that less mismatching information is required for rejection of a nontarget symbol than matching information is for acceptance of a target symbol.

In any case, caution is indicated by the small set of experimental points and rate $(v, u)$ values so far employed in conjunction with the above equations. The present development is largely meant to suggest potentially fruitful lines for theoretical and empirical investigation.

The $v_{n}^{+}, v_{n}^{-}$(or $u_{n}^{+}, u_{n}^{-}$) as functions of $n$ can be interpreted via feature testing mechanisms. When $v_{n}^{-} \neq v_{n}^{+}$, it could be due to different processing times on + and - comparisons or it might reflect different numbers of features required to establish a + or decision at the individual symbol level. With regard to information sufficient to compare a target with one of the multisymbol set, a - comparison requires only that a single mismatching feature be found, a + comparison only that a single feature match be found for a feature that is unique to the target. However, whether a $S$ is capable of being self-terminating at this level in either the + or - case is not known. Certainly, under either interpretation, the + and - feature comparison rate differing or the criterion number of features required for $a+$ or - comparison differing, the parallel (i.e., equal slope),+- curves imply that the difference depends on the number of items in the multisymbol set.

One of the conclusions that can be drawn from the above results in conjunction with other theoretical findings on parallel-serial identifiability is primarily negative: no one experiment is likely to decisively verify parallel or serial processing. In fact, even the profuse total body of data accumulated in the last decade on spatiotemporal processing of multisymbol displays and short-term memory does not appear to lead unequivocally to a narrow class of models.

On the positive side, even a single experiment can falsify a class of models; for example, Estes and Taylor's (1964) results falsified a fixed-sample size model and 
Sternberg's (1966) falsified a serial self-terminating model with equal, constant rates on + and - trials, and the above development has shown that a rather large class of models has been falsified by parallel + and curves containing underlying serial position effects. The class of models (e.g., the self-terminating models above plus exhaustive models with serial position effects taken out of comparison mechanisms) compatible with, say, parallel + and - curves should be susceptible to pairing down by successive integrated experiments. For instance, one of the central theses of the analysis above is that the + and - rates may depend on the target and noise sets, and on $n$. Then partitioning of, say, the class of self-terminating parallel and serial models according to possible underlying (micro) theories of how the rates should change (varying amount of feature overlap) with $n$ and subsequent appropriate experimental manipulation should lead to delimiting tests of the classes, as well as particular members of the classes of such models.

A closing point is that the processing modes (parallel vs serial, etc.) may well be different in ET than in LT designs, though the overall rates may appear quite comparable. Further, if ET comparisons do, indeed, take place in a visual store but LT in an acoustic store, then the features apposite for manipulation will differ respectively for the two.

\section{APPENDIX A}

The parallel RT prediction for each serial position in the + data is given by

$$
t_{n, i}^{+}=i_{0}^{+}+\frac{1}{v_{n, i}}
$$

where $t_{0}^{+}$represents the sum of residual latency components not associated with the comparison processing and is assumed to be additive with the latter and $n$ represents the number of comparisons ( $M$ or $D$ ) to be made. The parameter $v_{n, i}(i=1,2,3, \cdots, n-1, n: n$ $=1,2,3,4.5$ ) is the processing rate for Serial Position $\mathrm{i}$ when $\mathbf{n}$ sy mbols are being processed and is constant over time and number and order of other symbols processed for a given value of $\mathrm{n}$. There are 15 processing parameters and a residual RT parameter. There are 15 degrees of freedom in each of the + RT curves, but the $\mathrm{t}_{0}^{+}$parameter is needed to predict the nonzero intercept.

The expression for the overall self-terminating mean $\mathrm{RT}$ for the parallel model is given by

$$
R T_{n}^{+}=t_{0}^{+}+\frac{1}{n} \sum_{i=1}^{n} \frac{1}{v_{n, i}}
$$

and that for exhaustive RTs by

$$
\mathrm{RT}_{\mathrm{n}}^{-}=\mathrm{t}_{\mathrm{0}}^{-}+\frac{1}{v_{\mathrm{n}}} \sum_{\mathrm{i}=1}^{\mathrm{n}} \frac{1}{\mathrm{j}} \text {. }
$$

The latter formula. whith assumes homogeneous processing rates across serial positions, is a good deal less complex than one which assumes heterogeneous rates, given by

$$
\begin{aligned}
R T_{n}^{-}= & t_{0}^{-}+\sum_{i=1}^{n} \frac{1}{v_{n, i}} \sum_{i=1}^{n-1} \sum_{j=i+1}^{n} \frac{1}{\left(v_{i}+v_{j}\right)} \\
& +\sum_{i=1}^{n-2} \sum_{j=i+1}^{n-1} \sum_{k=j+1}^{n} \frac{1}{v_{i}+v_{j}+v_{k}} \\
& -\cdots+(-1)^{n-2} \sum_{i=1}^{n} \frac{1}{\sum_{\substack{j=1 \\
\neq \neq}}^{n} v_{j}}+(-1)^{n-1} \frac{1}{\sum_{k=1}^{n} v_{k}}
\end{aligned}
$$

It was found that both formulations gave similar predictions for means, and the simpler of the two was therefore used to predict the negative RTs.

Note that different $t_{0} \mathrm{~s}$ are allowed for the + and functions. This amounts to placing the more or less constant difference between the $+\mathrm{RT}$ and $-\mathrm{RT}$ curves outside the scanning mechanism [e.g., in response selection or response production, as Sternberg (1969) has suggested] .

In order to reduce the number of parameters in the serial model to that of the parallel, it was assumed that deviations from a strict left-to-right processing scan were uniform, e.g., if Serial Position 1 was not processed first, then any of the remaining $n-1$ positions were processed first with equal probability. Each display size then had $n-1$ probabilities, $p_{n, i}$, each being the probability that Serial Position i was processed jth: the remaining probabilities for the $i^{\text {th }}$ processing location were then given by

$$
\frac{1-p_{n, i}}{n-1}
$$

These assumptions, plus that of additivity of the residual $\mathrm{RT}$ components, lead to the following expressions:

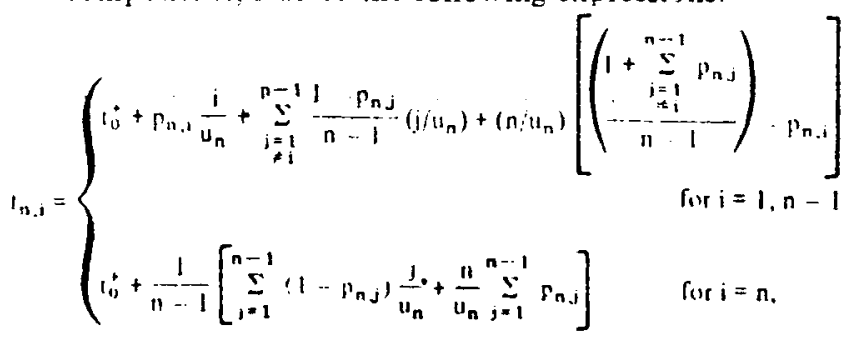

(Noke that we require $P_{n, i}+\sum_{j=i}^{n \cdots 1} \frac{1-P_{n j}}{n-1}=1-P_{n, a}$ )

$R T_{n}^{*}=10_{0}^{+}+\frac{n+1}{2} \frac{1}{u_{n}}$

$\operatorname{RT}_{n}^{-}=1_{0}^{-}+\frac{n}{u_{n}}$,

for the + serial position means. the orerall + RTs. and overall - RTs. respectively. for each value of $n=1.2 .3$. 4. 5 . The variahle $u_{n}$ is the processing rate for calch st mbol when $D$ ar $M$ equals n. 
The total class of mixed, exponential, serial models includes the class of unmixed, exponential, parallel models (the present serial and parallel models are members of these respective classes) in that for every such parallel model there is a serial model from the former class that is mathematically equivalent, but the converse is not true (for detailed definitions and discussion, see Townsend, 1971). Furthermore, although previous authors have implicitly or explicitly implied that an independent parallel model could not predict straight-line RT curves, this has been shown to be false (Townsend, 1972). This can be demonstrated by examination of the above mathematical expressions. Nevertheless, the present two models are not equivalent (e.g., the present serial model does not predict independence of processing on the separate symbols) and, in fact, at the level of the means, an independent parallel model can make predictions for the serial positions in + RTs that a serial model assuming a constant rate over serial positions cannot, even though the number of parameters is equal to the number of degrees of freedom in the data (Townsend, 1972). This can easily be illustrated by the case when $n=2$, for then

$$
\left.\begin{array}{rl}
t_{2,1}^{+} & =p \frac{1}{u_{2}}+(1-p) \frac{2}{u_{2}} \\
t_{2,2}^{+} & =p \frac{2}{u_{2}}+(1-p) \frac{1}{u_{2}}
\end{array}\right\} \quad \text { serial model }
$$

where $p$ is the probability that Serial Position 1 is processed first and $u_{2}$ is the processing rate for $n=2$ in the serial model and $\mathbf{v}_{2, i}(i=1,2)$ is the processing rate for Serial Positions 1 and 2, for $n=2$ in the parallel model. Solving for $p$ and $u_{2}$ in terms of the vs yields

$$
p=\frac{2 v_{2,1}-v_{2,2}}{v_{2,1}+v_{2,2}}, \quad u_{2}=\frac{3 v_{2,1}-v_{2,2}}{v_{2,1}+v_{2,2}} .
$$

If $t_{2,1}$ and $t_{2,2}$ are such that $v_{2,2}>2 v_{2,1}$, then unacceptable values for $p$ are determined, and the serial model is falsified. When $t_{0}^{+}$is included, this demonstration involves considering more than one value of $n$, but an analogous result obtains. This shows that the parallel model has to fit the data but the serial model may not.

\section{APPENDIX B}

It is sufficient, as well as intuitively instructive, to show that the functions are unable to maintain an equal slope even from $n=1$ to $n=2$. The + processing times for $n=1$ and $n=2$ are:

$$
\begin{aligned}
\mathrm{RT}_{1}^{+}-\mathrm{t}_{0}^{+}= & \mathrm{E}_{1}\left(\mathrm{t}^{+}\right), \\
\mathrm{RT}_{2}^{+}-\mathrm{t}_{0}^{+}= & \frac{1}{2}\left\{\mathrm{pE}\left(\mathrm{t}_{11}^{+}\right)+(1-\mathrm{p})\left[\mathrm{E}\left(\mathrm{t}_{21}^{-}\right)+\mathrm{E}\left(\mathrm{t}_{12}^{+}\right)\right]\right\} \\
& \left.\left.+\frac{1}{2}\right\}(1-\mathrm{p}) \mathrm{E}\left(\mathrm{t}_{21}^{+}\right)+\mathrm{p}\left[\mathrm{E}\left(\mathrm{t}_{11}^{-}\right)+\mathrm{E}\left(\mathrm{t}_{22}^{+}\right)\right]\right\} ;
\end{aligned}
$$

and those for the - RTs are:

$$
\begin{aligned}
\mathrm{RT}_{1}^{-}-\mathrm{t}_{0}^{-} & =\mathrm{E}_{1}\left(\mathrm{t}^{-}\right) \\
\mathrm{RT}_{2}^{-}-\mathrm{t}_{0}^{-} & =\mathrm{p}\left[\mathrm{E}\left(\mathrm{t}_{11}^{-}\right)+\mathrm{E}\left(\mathrm{t}_{22}^{-}\right)\right] \\
& +(1-\mathrm{p})\left[\mathrm{E}\left(\mathrm{t}_{12}^{-}\right)+\mathrm{E}\left(\mathrm{t}_{21}^{-}\right)\right] .
\end{aligned}
$$

By showing that $\left(\mathrm{RT}_{1}^{-}\right)-\left(\mathrm{RT}_{1}^{+}\right) \neq\left(\mathrm{RT}_{2}^{-}\right)-\left(\mathrm{RT}_{2}^{+}\right)$, we shall have succeeded in our proof. But, gathering terms together in a way that we can use the equal average increment assumptions, we obtain

$$
\begin{aligned}
& \left.\left.R T_{2}\right)-\left(R T_{2}^{*}\right)=\frac{1}{2}|P| E\left(1_{11}^{-}\right)+E\left(1_{2}^{-}\right)\right]+(1-p)\left\{\left[:\left(1_{12}^{-}\right)+E\left(1_{21}^{-}\right)\right\} \mid\right.
\end{aligned}
$$

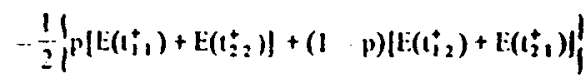

$$
\begin{aligned}
& +\frac{1}{2}\left\{p\left(E\left(t_{11}^{-}\right)+E\left(t_{22}^{-}\right)|+(1-p)| E\left(t_{12}^{-}\right)+E\left(t_{12}^{-}\right)\right) \mid\right.
\end{aligned}
$$

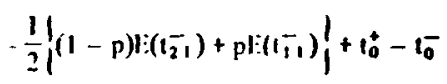

$$
\begin{aligned}
& \left.\left.=E\left(1_{1}\right)-E\left(i_{1}^{*}\right)+\frac{1}{2}\right\}^{\mathrm{pE}\left(1_{22}^{-}\right)+(1-p) E\left(1_{12}\right)}\right\}^{1}+t_{0}^{*}-t_{0}^{-} \\
& \equiv E\left(t_{i}^{-}\right)-E\left(t_{i}^{*}\right)+t_{0}^{*}-t_{0}^{-},
\end{aligned}
$$

that is, equality implies that

$$
\frac{1}{2}\left[p E\left(t_{22}^{-}\right)+(1-p) E\left(t_{12}^{-}\right)\right] \equiv 0,
$$

which in turn implies that $\mathrm{E}\left(\mathrm{t}_{22}\right)=\mathrm{E}\left(\overrightarrow{\mathrm{t}_{12}}\right)=0$; that is, any - symbol processed second is processed infinitely fast, which is absurd. Thus, this class of models cannot predict equal RT slopes for + and - trials. Furthermore, the error term

$$
\frac{1}{2}\left[\mathrm{pE}\left(\mathrm{t}_{22}^{-}\right)+(1-\mathrm{p}) \mathrm{E}\left(\mathrm{t}_{12}^{-}\right)\right],
$$

may be substantial. For example, if

$$
\mathrm{p}=\frac{1}{2}, \mathrm{E}\left(\mathrm{t}_{22}^{-}\right)=\mathrm{E}\left(\mathrm{t}_{22}^{-}\right)=20 \mathrm{msec},
$$

which is about the average time increment per letter here, then it amounts to a $10-\mathrm{msec}$ deviation from slope equality (large relative to the average standard error of $5.32 \mathrm{msec}$ ). 


\section{REFERENCES}

A tkinson, R. C., Holmgren, J. E., \& Juola, J. F. Processing time as influenced by the number of elements in a visual display. Perception \& Psychophysics, 1969, 6, 321-326.

Bamber, D. Reaction times and error rates for "same"-"different" judgments of multidimensional stimuli. Perception \& Psychophysics, 1969, 6, 169-174.

Brand, J. Classification without identification in visual search. Quarterly Journal of Experimental Psychology, 1971, 23, 178-186.

Briggs, G. E., \& Swanson, J. M. Retrieval times as a function of memory ensemble size. Quarterly Journal of Experimental Psychology, 1969, 21, 185-191.

Briggs, G. E., \& Swanson, J. M. Encoding, decoding, and central functions in human information processing. Journal of Experimental Psychology, 1970, 86, 296-308.

Burrows, D., \& Murdock, B. B., Jr. Effects of extended practice on high-speed scanning. Journal of Experimental Psychology, $1969,82,231-237$.

Clifton, C., \& Birenbaum, S. Effects of serial position and delay of probe in a memory scan task. Journal of Experimental Psychology, 1970, 86, 69-76.

Corballis, M. C. Serial order in recognition and recall. Journal of Experimental Psychology, 1967, 74, 99-105.

Egeth, H., Jonides, J., \& Wall, S. Parallel processing of multi-element displays. Cognitive Psychology, 1972, 3, 674-698.

Estes, W, K., \& Taylor, H. A. A detection method and probabilistic model for assessing information processing from brief visual displays. Proceedings of the National Academy of Science, 1964, 52, 446-454.

Estes, W. K., \& Taylor, H. A. Visual detection in relation to display size and redundancy of critical elements. Perception \& Psychophysics, 1966, 1, 9-16.

Estes, W. K., \& Wessel, D. L. Reaction time in relation to display size and correctness of response in forced-choice visual signal detection. Perception \& Psychophysics, 1966, 1, 369-373.

Gardner, G. T. Spatial processing characteristics in the perception of brief visual arrays. Technical Report No. 23, Human Performance Center, University of Michigan, Ann Arbor, 1970.

Klatzky, R. L., \& Atkinson, R. C. Memory scans based on alternative test stimulus representations. Perception \& Psychophysics, 1970, 8, 113-117.

Klatzky, R. L., Juola, J. F., \& Atkinson, R. C. Test stimulus representation and experimental context effects in memory scanning. Journal of Experimental Psychology, 1971, 87, 281-288.

Klemmer, E. T. Does recoding from binary to octal improve perception of binary patterns? Journal of Experimental Psychology, 1964, 67, 19-21.

Morin, R. E., DeRosa, D. V., \& Stultz, V. Recognition memory and reaction time. Acta Psychologica, 1967, 27, 298-305.

Murdock, B. B., Jr. A parallel-processing model for scanning. Perception \& Psychophysics, 1971, 10, 289-291.

Nickerson, R. S. Response times with a memory dependent decision task. Journal of Experimental Psychology, 1966, 72, 761-769.

Norman, D. A. Acquisition and retention in short-term memory. Journal of Experimental Psychology, 1966, 72, 369-381.

Pollack, I., \& Johnson, L. B. Memory span with efficient coding procedures. American Journal of Psychology, 1965, 78, 609-614.

Sperling, G. The information available in brief visual presentations. Psychological Monographs, 1960, 74(11, Whole No. 498).

Sternberg, S. High-speed scanning in human memory. Science, $1966,153,652-654$.

Sternberg, S. The discogvery of processing stages: Extensions of
Donder's method. Acta Psychologica, 1969, 30, 276-315.

Sternberg, S. Scanning a persisting visual image versus a memorized list. Paper presented at the Annual Meeting of the Eastern Psychological Association, 1967.

Swanson, J. M., \& Briggs, G. E. Information processing as a function of speed versus accuracy. Journal of Experimental Psychology, 1969, 81, 223-229.

Theios, J., Smith, P. G., Haviland, S. E., Traupmann, J., \& Moy, M. C. Memory scanning as a serial self-terminating process. Journal of Experimental Psychology, 1973, 97, 323-336.

Townsend, J. T. A note on the identifiability of parallel and serial processes. Perception \& Psychophysics, 1971, 10, 161-163.

Townsend, J. T. Some results concerning the identifiability of parallel and serial processes. British Journal of Mathematical \& Statistical Psychology, 1972, 25, 168-199.

Weaver, S., McLaughlin, J. P., \& Branca, A. A. Retrieval processes in recent memory. Proceedings of the 78th Annual Convention of the American Psychological Association, 1970 , $5,95-96$.

Wingfield, A., \& Bolt, R. A. Memory search for multiple targets. Journal of Experimental Psychology, 1970, 85, 45-50.

Wingfield, A., \& Branca, A. A. Strategy in high-speed memory search. Journal of Experimental Psychology, 1970, 83, 63-67.

\section{NOTES}

1. Some notable exceptions are certain conditions reported by Egeth, Jonides, and Wall (1972), in which they found flat (ET) functions.

2. Since the aim was to see if either a fairly general, but typical, parallel or serial model could predict both the $+\mathrm{RT}$ and the - RT data, the number of parameters in the two types of models was allowed to be quite large in fitting the + RT data. Serial position data and (therefore) overall means were fit in the + RT data. As will be seen, this generality did not prevent the two models from failing miserably to fit the - RT data. The very generality of the two models strengthens our falsifying conclusions regarding their predictive ability.

3. "Independent" is used here to mean statistical independence of processing among symbols. Thus, if symbols " $a$ " and " $b$ " were undergoing parallel processing and it was further known that " $b$ " had been completed by time T, say, this (conditional) information would have no bearing on the likelihood that " $a$ " had also been completed by time T. The term, "independence," is also used to indicate a functional independence of (typically parallel) processing rates from the number of objects (e.g., symbols) being processed. The latter sense of independence signifies that the "capacity" or "energy" resources of a system are of a relatively unlimited nature (Gardner, 1970; Townsend, 1971).

4. The parameters were all obtained via method of moments estimation, with the exception of the $t_{0}$ variables, which reflect residual latency mechanisms. For exhaustive processing, $t_{0}$ was estimated as the zero intercept of the function, and for self-terminating processing, $t_{0}$ was the intercept minus the slope. The zero intercepts used were the intercepts of the best-fitting straight lines through the functions, except for the LT positive data, where this intercept was greater than the mean RT for $M=1$. A more reasonable estimate $(530.6 \mathrm{msec})$ was obtained by assuming that processing rates were equal to $\mathrm{M}=1,2$.

5. It should be noted that a fortiori the class of parallel models that is nonidentifiable with respect to the present class of serial models also is falsified by parallel (equal slope) + and $-R T$ functions.

6 . The serial model, as well as the parallel model below, do not depend on dual mechonisms but only on the precept that and + comparisons may proceed at different rates. In fact, the 
serial model seems more compatible with a unary processing mechanism, although the parallel model could be intuitively associated with either one.

7. Lappin (personal communication) has suggested the possibility that some type of organizing process (e.g., Gestalt) might yield such a speeding up of processing. Note, however, that processing has to become faster on the arerage for a larger fixed number of symbols. This concept must be kept distinct from the possible speeding up or slowing down that might take place within a trial from the rate on the first symbol to that on the last processed on that trial. The latter could occur even though the overall average rate of processing was constant as a function of $n$.

(Received for publication February 18, 1973: accepted March 3. 1973.) 